\title{
COMMENTARY
}

\section{How do we know when patients sleep properly or why they do not?}

\author{
Folke Sjöberg ${ }^{* 1,2}$ and Eva Svanborg ${ }^{2,3}$ \\ See related research by Elliot et al., http://ccforum.com/content/17/2/R46
}

\begin{abstract}
The importance of adequate sleep for good health and immune system function is well documented as is reduced sleep quality experienced by ICU patients. In the previous issue of Critical Care, Elliot and co-workers present a well done, largest of its kind, single-center study on sleep patterns in critically ill patients. They base their study on the 'gold standard', the polysomnography technique, which is resource demanding to perform and often difficult to evaluate. The results are especially interesting as the authors not only used polysomnography in a large sample but also, in contrast to others, excluded patients with prior sleep problems. They also recorded patients' subjective sleep experiences in the ICU and thereafter in the ward (validated questionnaires) with simultaneous data collection of factors known to affect sleep in the ICU (mainly treatment interventions, light and sound disturbances). Interestingly, but not surprisingly, sleep was both quantitatively and qualitatively poor. Furthermore, there seemed to be little or no improvement over time when compared to earlier studies. This study stresses the magnitude of the sleep problem despite interventions such as earplugs and/or eyeshades. Sound disturbance was found to be the most significant but improvable factor. The study highlights the challenge and the importance of evaluating sleep in the critical care setting and the present need for alternative methods to measure it. All that in conjunction can be used to solve an important problem for this patient group.
\end{abstract}

*Correspondence: folke.sjoberg@liu.se

2Department of Clinical and Experimental Medicine, Linköping University, Linköping, 58185 Sweden

Full list of author information is available at the end of the article
The role of sleep in general health is well known. Poor sleep predicts cardiovascular disease [1], and increases oxidative stress [2]. It also increases pro-inflammatory cytokines and worsens subjective health [3]. Furthermore, which may be especially important in ICU patients, short sleep duration increases the susceptibility to infections [4]. It is also well known that many critically ill patients experience sleep disruptions, which is assumed to have health implications [5]. Important also is the fact that the ICU population as such is a heterogeneous group burdened with increased rates of co-morbidities [6]. Preexisting diseases - that is, poor health - comprise a wellknown factor that affects sleep during 'normal' conditions and sleep patterns may be thought to further deteriorate during periods of critical illness and affect health-related quality of life [7]. The effect of such induced poor sleep may therefore result in further health difficulties. Such complicating background factors may have implications for investigations, such as that by Elliot and co-workers in the previous issue of Critical Care [8], as it is difficult to know the 'normal' sleep pattern of any patient and, consequently, in sleep research examining hospital populations, the choice of control group is a challenge.

Significant efforts are continuously being directed to assess and combat organ dysfunction and failure in critical illness. Among these has been a recent focus on the brain supported by results from the anesthesia perspective where cognitive dysfunction has been registered after general anesthesia and surgery or heart surgery. Concomitantly, a hot topic in critical care has been the issue of delirium, possibly caused by central nervous system failure and/or as a consequence of sedation techniques, other drugs or even exhaustion due to sleep deprivation, possibly caused by direct practical carerelated issues $[9,10]$. A significant portion of critically ill patients are furthermore sedated, not uncommonly as a substitute for not being able to sleep otherwise. This underlines the importance of sleep scoring as a part of overall brain function monitoring and to assess when there is need for supportive measures. Still, brain function assessment and surveillance in the ICU setting, both for clinical and scientific purposes, is difficult due to 
a shortage of relevant and practical methods to use [11]. For clinical use practical techniques are sought that are validated and that are specific for the scientific counterparts. Neither of these are available today, as exemplified by, for example, the low sensitivity of the best recommended assessment tool for delirium [12]. Furthermore, besides the strictly practical difficulties seen in the ICU setting, there is also a lack of knowledge and consensus on how to assess cognitive functions in this patient group, who often in parallel are also affected by pharmacological agents.

Elliot and colleagues [8] contribute significantly to an important aspect of brain function monitoring by examining sleep patterns. These we know have very significant health implications. The authors are to be congratulated on their high level of ambition to gain knowledge regarding sleep patterns in the ICU by performing a large study using good methodology including the use of polysomnography (the 'gold standard') combined with adequate, validated patient questionnaires and simultaneous data collection of data depicting factors known to affect sleep in the ICU.

Furthermore, previous studies have rarely collected data on the patient perception of sleep quality and the potential causes of sleep disturbing factors, important factors that have also been included in the present study. The study shows clearly, as may be expected, that the patients included in the study experience poor sleep and that this issue should be further examined and improvement interventions developed. Another and possibly even more important issue that the authors stress in their article is the demanding nature and practical difficulties known for this methodology. It needs then to be appreciated that although 656 patients satisfied inclusion criteria, only 53 patients (less than 10\%) were in the end included and examined. Many drop outs are due to different methodological issues, that is, difficulties in evaluating sleep in situations where, for example, sleep patterns prior to ICU are already poor or patients are neurologically impaired. This unfortunately reduces the generalizability of the results. In delirium or cognitive dysfunction, electroencephalography frequencies are often slow already in wakefulness, which makes assessment of sleep stages difficult. Many pharmacological agents (for example, benzodiazepines, morphine) in themselves also change the electroencephalogram and/or sleep patterns. Absence of information concerning medication and presence of pain (also very important when it comes to degree of sleep disturbance) are minor flaws in the present report. An obvious practical shortcoming complicating sleep evaluations - compared to, for example, diagnosing delirium or cognitive dysfunction - is that many examining procedures for this purpose may in themselves affect sleep if not done in specialized laboratories.

\section{What is the take home message?}

There are several. Firstly, in the group of patients that can be properly examined there are significant quantitative and qualitative sleep disturbances. Secondly, as of today the clinical routines to optimize sleep in the critical care setting may still not be fully optimized and sound and care intervention disturbances are the ones to address first. Thirdly, sleep monitoring and research in the critical care setting are technically difficult due to a lack of easy to use and reliable methods. With our current methodologies and knowledge few patients can be properly examined for various reasons and this reduces generalizability of the conclusions. Our knowledge today regarding sleep, an important brain function, is based mainly on a minority of patients and this may only reveal the top of a large iceberg.

\section{Conclusion}

The study by Elliot and co-workers is a well performed study on the important topic of sleep, which is closely related to brain function, dysfunction and failure. Among several important findings, it underlines the need for new monitoring and research tools in order to better understand sleep for this patient group and to develop care improvement strategies.

\section{Competing interests \\ The authors decalre that they have no competing interests}

\section{Author details}

'The Burn Center, Departments of Hand, Plastic Surgery and Intensive Care, Linköping County Council, Linköping, 58185 Sweden. ${ }^{2}$ Department of Clinical and Experimental Medicine, Linköping University, Linköping, 58185 Sweden. ${ }^{3}$ Department of Clinical Neurophysiology, Linköping County Council, Linköping, 58185 Sweden.

\section{Published: 15 May 2013}

\section{References}

1. Cappuccio FP, Cooper D, D’Elia L, Strazzullo P, Miller MA: Sleep duration predicts cardiovascular outcomes: a systematic review and meta-analysis of prospective studies. Eur Heart J 2011, 32:1484-1492.

2. Everson CA, Laatsch CD, Hogg N: Antioxidant defense responses to sleep loss and sleep recovery. Am J Physiol Regul Integr Comp Physiol 2005, 288:R374-383.

3. Vgontzas AN, Zoumakis E, Bixler EO, Lin HM, Follett H, Kales A, Chrousos GP: Adverse effects of modest sleep restriction on sleepiness, performance, and inflammatory cytokines. J Clin Endocrinol Metab 2004, 89:2119-2126.

4. Cohen S, Doyle WJ, Alper CM, Janicki-Deverts D, Turner RB: Sleep habits and susceptibility to the common cold. Arch Intern Med 2009, 169:62-67.

5. Freedman NS, Gazendam J, Levan L, Pack Al, Schwab RJ: Abnormal sleep/ wake cycles and the effect of environmental noise on sleep disruption in the intensive care unit. Am J Respir Crit Care Med 2001, 163:451-457.

6. Orwelius L, Nordlund A, Nordlund P, Simonsson E, Bäckman C, Samuelsson A, Sjöberg F: Pre-existing disease: the most important factor for health related quality of life long-term after critical illness: a prospective, longitudinal, multicentre trial. Crit Care 2010, 14:R67.

7. Orwelius L, Nordlund A, Nordlund P, Edéll-Gustafsson U, Sjöberg F: Prevalence of sleep disturbances and long-term reduced health-related quality of life after critical care: a prospective multicenter cohort study. Crit Care 2008, 12:R97.

8. Elliott R, McKinley S, Cistulli P, Fien M: Characterisation of sleep in intensive care using 24-hour polysomnography: an observational study. Crit Care 2013, 17:R46. 
9. Pisani MA, Kong SY, KasI SV, Murphy TE, Araujo KL, Van Ness PH: Days of delirium are associated with 1-year mortality in an older intensive care unit population. Am J Respir Crit Care Med 2009, 180:1092-1097.

10. van Eijk MM, Slooter AJ: Duration of ICU delirium, severity of the underlying disease, and mortality. Am J Respir Crit Care Med 2010, 181:419-420; author reply 420-421.

11. Tempelhoff R, Yoder J: Monitoring the brain: lack of tools or lack of will? Crit Care Med 2008, 36:1983-1984.
12. Vasilevskis EE, Girard TD, Ely EW: The bedside diagnosis of ICU delirium: specificity is high, let's optimize sensitivity. Am J Respir Crit Care Med 2012, 185:107-108; author reply 108

doi:10.1186/cc12614

Cite this article as: Sjöberg F, Svanborg E: How do we know when patients sleep properly or why they do not? Critical Care 2013, 17:145. 